EPJ Web of Conferences 71, 00027 (2014)

DOI: 10.1051/epjconf/20147100027

(C) Owned by the authors, published by EDP Sciences, 2014

\title{
The CMS Masterclass and Particle Physics Outreach
}

Kenneth Cecire ${ }^{1 \mathrm{a}}$, Marjorie Bardeen ${ }^{2}$, and Thomas McCauley ${ }^{1}$

\author{
${ }^{1}$ Department of Physics, University of Notre Dame, 225 Nieuwland Science Hall, Notre Dame, \\ Indiana 46556 U.S.A \\ ${ }^{2}$ Fermi National Accelerator Laboratory, Education Office, MS 226, P.O. Box 500, Batavia, Illinois \\ 60510 U.S.A.
}

\begin{abstract}
The CMS Masterclass enables high school students to analyse authentic CMS data. Students can draw conclusions on key ratios and particle masses by combining their analyses. In particular, they can use the ratio of $\mathrm{W}^{+}$to $\mathrm{W}^{-}$candidates to probe the structure of the proton, they can find the mass of the $\mathrm{Z}$ boson, and they can identify additional particles including, tentatively, the Higgs boson. In the United States, masterclasses are part of QuarkNet, a long-term program that enables students and teachers to use cosmic ray and particle physics data for learning with an emphasis on data from CMS.
\end{abstract}

\section{Introduction}

The CMS Masterclass [1] is one prominent example of the use of authentic particle physics data to bring compelling physics to high schools. CMS, the Compact Muon Solenoid [2], is one of two general-purpose experiments at the Large Hadron Collider (LHC) at CERN, Geneva, Switzerland. During International Masterclasses 2013 [3], 50 masterclass institutes in 17 countries offered the CMS Masterclass, roughly $1 / 4$ of the total in 2013. To examine the CMS Masterclass, then, is to get some sense of how students look at the data.

There are two measurements available in the CMS Masterclass: the $\mathrm{J} / \Psi$ measurement and the WZ measurement. The latter is used in International Masterclasses while the former is reserved for individual high schools. Successful examples of use of the $J / \Psi$ measurement in the U.S. are the masterclasses done locally at St. Joseph High School by Loughran et al [4] and at the Trinity Schools by Mooney et al [5]. However, these are not in the scope of this paper. In 2014, the WZ measurement will become the WZH measurement with the addition of a small number of diphoton Higgs candidate events. The term "CMS Masterclass" will refer to the WZ or WZH measurement in this paper. We will examine some of the physics that students explore in the CMS Masterclass, how they go about making their measurement, and how results are handled. The overall structure of masterclasses is referred to but not discussed in detail, as this is covered elsewhere [6].

There is additional work in particle physics and cosmic ray physics education and outreach, much of it based on the use of large (for high school) sets of authentic data. Much has been done in this regard by the QuarkNet collaboration [7] in the U.S.; we will survey this work and draw conclusions.

\section{CMS Masterclass}

The CMS Masterclass is built around 1900 events (to be expanded to 3000 in 2014) from the LHC 7 and $8 \mathrm{TeV}$ runs. The data consists primarily of single lepton plus neutrino (W candidate) events, dilepton events (called $\mathrm{Z}$ candidates in the measurement but not limited to $\mathrm{Z}$ bosons), and background. There are a few 4-lepton Higgs candidates (called ZZ candidates in the 2013 measurement). In 2014, a small number of diphoton Higgs candidates will be added; these and the former 4-lepton Higgs

\footnotetext{
${ }^{\mathrm{a}}$ Corresponding author: kecire@nd.edu
}

This is an Open Access article distributed under the terms of the Creative Commons Attribution License 2.0, which permits unrestricted use, distribution, and reproduction in any medium, provided the original work is properly cited. 
candidates will all be labeled "Higgs candidates" in 2014, and the "ZZ" designation will be dropped as a label.

Each CMS Masterclass Institute is a one-day event characterized by a series of activities, not always in the same order:

- (Classroom preparation before day of masterclass)

- Registration/Greetings/Introduction

- Physicist presentation on CMS physics

- Lab tour

- Teacher or physicist presentation on how to analyse data

- Student pair analysis of 100 events per pair at computers using visual event display

- Physicist-led discussion of combined results for the institute

- Videoconference with other CMS masterclass institutes and moderators at CERN or Fermilab

- Wrap-up.

Classroom preparation (first step in parentheses) is not done for all masterclasses. It is most common in the U.S. due to the influence of QuarkNet.

\subsection{W physics and probing proton structure at a student level}

\subsection{1 $W$ boson identification in LHC}

$\mathrm{W}^{+}$and $\mathrm{W}^{-}$bosons are produced directly in large quantities in LHC proton collisions. They promptly decay. In the CMS masterclass, the observed decays are

$$
\begin{aligned}
& \mathrm{W}^{ \pm} \rightarrow \mathrm{e}^{ \pm} v \\
& \mathrm{~W}^{ \pm} \rightarrow \mu^{ \pm} v .
\end{aligned}
$$

Students characterize W decays as either "electron" or "muon" according to the tracks observed in the iSpy-online event display [8]. Short tracks (color-coded yellow) that do not go beyond the tracker and end in an energy deposit in the electromagnetic calorimeter are said to represent electrons or positrons. Longer tracks (color-coded red) with few or no energy deposits that extend to the CMS muon system are said to be muons. Neutrinos are not detected by CMS but show as "missing $\mathrm{E}_{\mathrm{t}}$ "; that is, the transverse momenta of all of the detected particles in an event are added and where there is a non-zero sum, it is inferred that the equal and opposite vector represents the transverse momentum of a neutrino. 


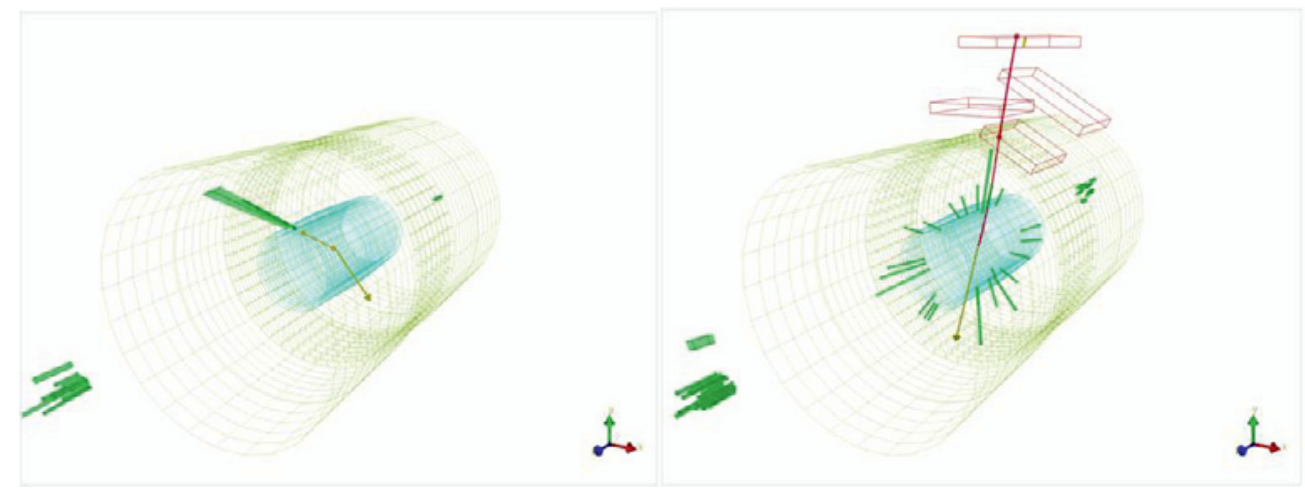

Figure 1. Candidate $\mathrm{W}$ decays in iSpy-online. A decay to an electron and a neutrino (left) and a decay to a muon and a neutrino (right).

For each $\mathrm{W}$ candidate event, students must identify it as such and record whether it is an electron event or a muon event. Students must also identify, where possible, the charge of the $\mathrm{W}$ boson parent particle. They do this by looking at the event in an $\mathrm{x}-\mathrm{y}$ view (i.e. viewed along the z-axis) of the event and determining whether the charged lepton track curves clockwise (positive charge) or anticlockwise (negative charge) in the magnetic field inside the CMS solenoidal magnet. A helpful feature of iSpy-online is that event images can be manipulated: they can be rotated or snapped to $\mathrm{x}-\mathrm{y}, \mathrm{y}-\mathrm{z}$, or $\mathrm{X}-\mathrm{Z}$ views. The display view can also be panned and zoomed.

Identifying $\mathrm{W}$ events, distinguishing between electrons and muons, and determining the charge using the event display can be very effective. An analysis of the results obtained by a group of highschool students from Annecy, France that participated in a masterclass at CERN found that students were able to determine that an event is a $\mathrm{W}$ event with $95 \%$ efficiency. Of those events that they estimated to be a $\mathrm{W}$, they were able to determine the charge (from electron or muon) for $92 \%$ of the events (if not, they marked them as "W candidate" with no charge specified). Of those events where they were able to determine the charge, they were able to determine it correctly with $96 \%$ efficiency.

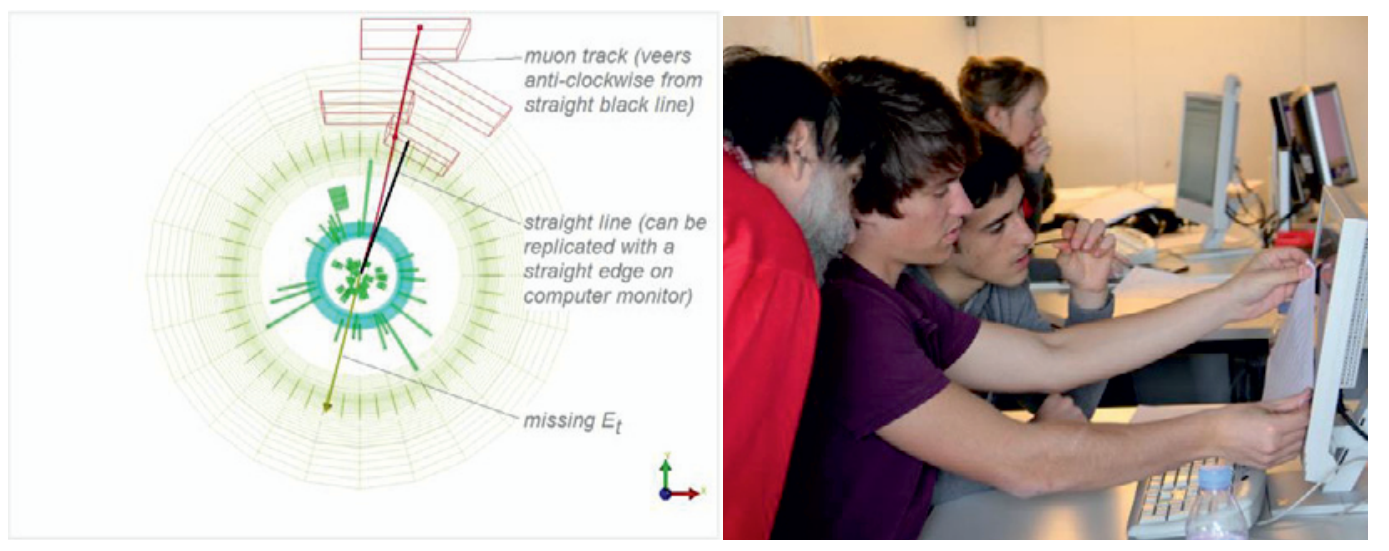

Figure 2. Determination of $\mathrm{W}^{-}$boson charge using the curvature of the track of its daughter antimuon (left). For electrons and positrons, the task is more difficult but can be accomplished by zooming in. Students often use their information sheet as a straight edge in charge determination (right). 


\subsubsection{Probing the proton from $W$ boson production}

When protons collide, the $\mathrm{W}$ boson can be produced from quark-quark interactions. From charge conservation, this must come from a quark-antiquark interaction. For example, an up quark (charge $+2 / 3$ ) combines with an antidown quark (charge $+1 / 3$ ) to make a $\mathrm{W}^{+}$boson. In the same way, a down quark (charge $-1 / 3$ ) can combine with an antiup quark (charge $-2 / 3$ ) to form a $\mathrm{W}^{-}$boson. The valence quarks of the proton are 2 up quarks and 1 down quark: the antiquarks must come from the "sea" of virtual quarks in the proton, which have an even chance of being a negative or a positive match for the valence up and down quarks. That would work out to a $2: 1$ ratio of $\mathrm{W}^{+}$to $\mathrm{W}^{-}$. However, a sea quark can encounter a sea antiquark-even chances all around, working out to a 1:1 ratio. The actual result in CMS is somewhere in-between: the measurement at $7 \mathrm{TeV}$ with $36 \mathrm{pb}^{-1}$ integrated luminosity is very close to 1.42 [9]. The result from CMS masterclasses in March 2013 is closer to 1.22 (seen in Figure 4). The lower ratio in the masterclass is by design. The absolute number of $\mathrm{W}$ candidate events in the samples is determined from simple counting. There are no corrections for efficiency and acceptance as in the published CMS result. These corrections are different for muons and electrons so if students were to look at the ratios for muons only and for electrons only they would see that the electron ratio will be closer to 1.2 and the muon closer to the actual number as determined by CMS.

\subsection{Further CMS masterclass results}

\subsubsection{Dilepton events}

Students also search for dilepton events in the CMS Masterclass. These are initially labelled " $Z$ boson candidates" based on being $\mathrm{e}^{+} \mathrm{e}^{-}$or $\mu^{+} \mu^{-}$events, regardless of calculated invariant mass, which is provided. Students place these events in a mass plot that runs in $2 \mathrm{GeV}$ intervals up to $135 \mathrm{GeV}$. (This is done by the expedient of rounding any mass to the nearest odd number.) After combining data in their institute, students discover bumps not only at the $\mathrm{Z}$ mass but also at roughly $3 \mathrm{GeV}$ and 9-11 $\mathrm{GeV}$ so that they can, with the help of a physicist, identify $\mathrm{J} / \Psi$ and $\mathrm{Y}$ peaks.

\subsubsection{Higgs candidates}

In the 2013 CMS Masterclass, there are three candidate Higgs events in the form of decays to 4 leptons. These are repeated so that each pair of students has a chance to find one. They are initially reported as "ZZ candidates" as they are presumably produced in the decay chain

$$
\mathrm{H} \rightarrow \mathrm{ZZ} * \mathrm{I}^{+} \mathrm{1}^{-1} \mathrm{1}^{-}
$$

Students are given the 4-lepton invariant mass of each of these, which they put in the mass plot. It produced a small bump, hard to distinguish from background, so that students have a "hint" of the Higgs.

The 2014 CMS Masterclass will also have 10 diphoton Higgs candidates. Two will be in each set of 100 events. Both forms of Higgs candidate (4-lepton and diphoton) will be designated as such and will go into the mass plot.

\subsubsection{Student results in 2013}

In the 2013 CMS Masterclass, students were able to identify $\mathrm{J} / \Psi, \mathrm{Y}$ and $\mathrm{Z}$ peaks along with a Higgs bump. They found $\mathrm{W}^{+}$to $\mathrm{W}^{-}$ratios that varied depending on their interpretation of results and the 
number of events they analysed in their institute but which had a central value of approximately 1.22. They also found interesting "zoo" events that were hard to categorize at all.

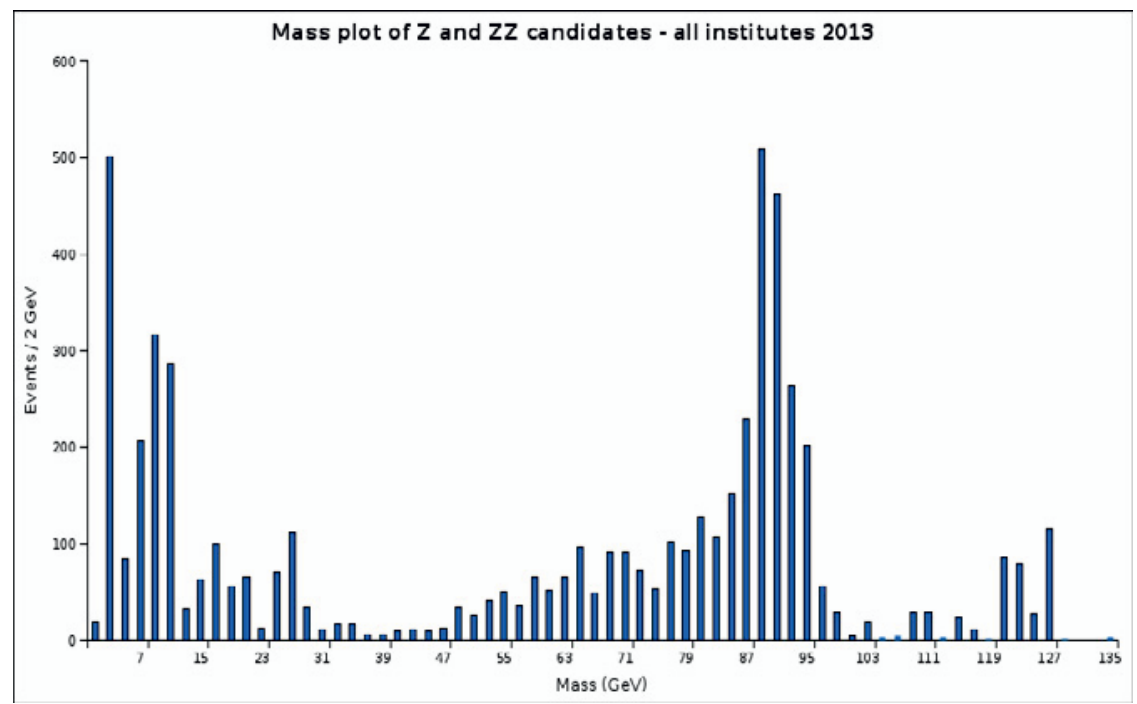

Figure 3. Mass plot from all CMS Masterclass Institutes in March 2013.

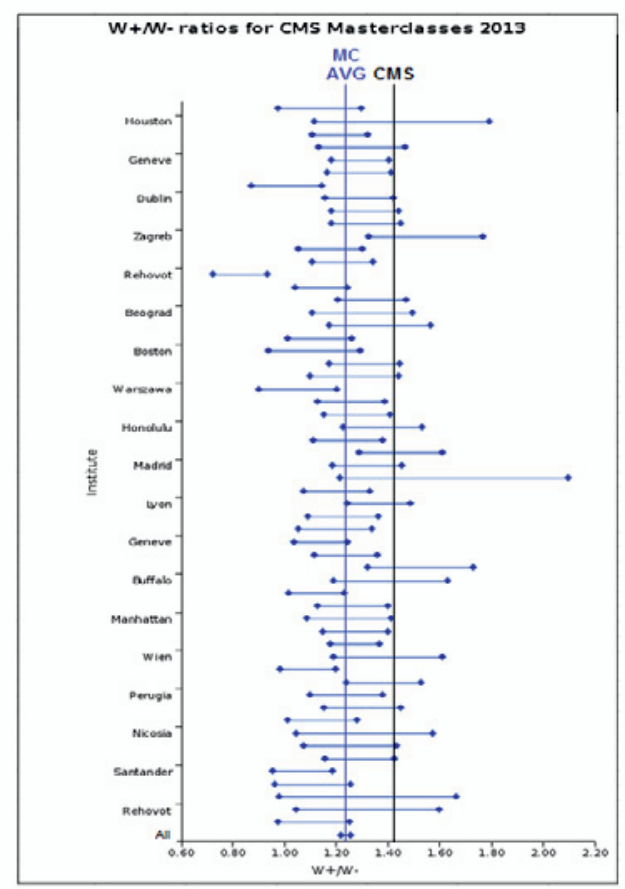

Figure 4. $\mathrm{W}^{+} / \mathrm{W}^{-}$results for all CMS Masterclass Institutes in March 2013. 


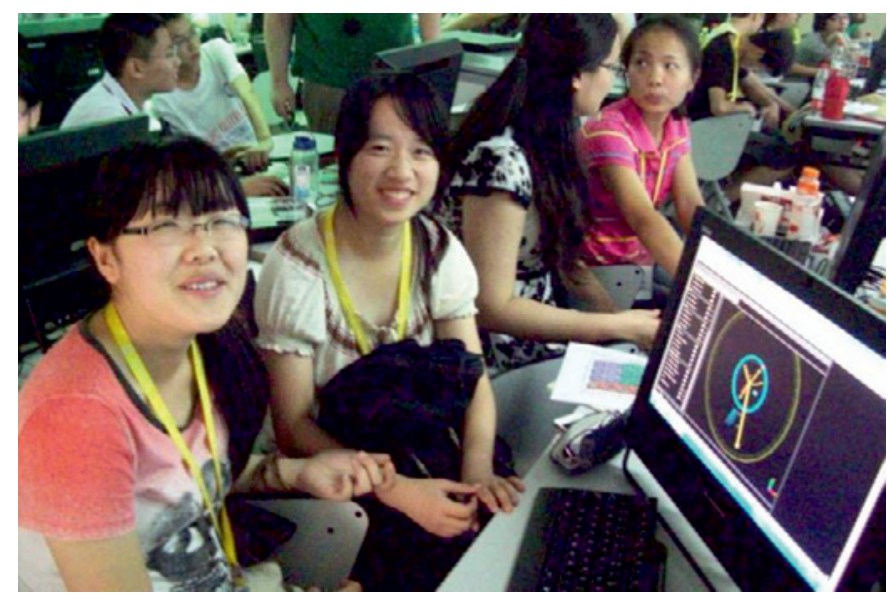

Figure 5. Students in Beijing, China showing a "zoo" event they discovered in a masterclass.

\section{Particle Physics Outreach and Data in the U.S. and beyond}

The CMS Masterclass is a leading example of a rich portfolio of student investigations and activities that are emerging worldwide, particularly in the U.S. through QuarkNet. These exist in an environment of shared work in learning communities of physics teachers and physicists.

\subsection{The QuarkNet collaboration}

QuarkNet is a professional development education program primarily for high school physics teachers funded by the U.S. National Science Foundation and formerly the U.S. Department of Energy. Its aims are to reform physics education through the methods and the content of experimental particle physics and to bring authentic particle physics data, especially from the LHC, to high school students and teachers.

To accomplish these aims, QuarkNet is organized into QuarkNet centers at universities or laboratories supported by a national infrastructure. Each center strives to be a learning community with two or more physicist mentors and up to ten high school physics teachers. In addition, a national staff assists the centers and actively works on building the QuarkNet data portfolio.

Since particle physics is built on international collaborations, QuarkNet has moved forward by building its own alliances with physicists and educators worldwide.

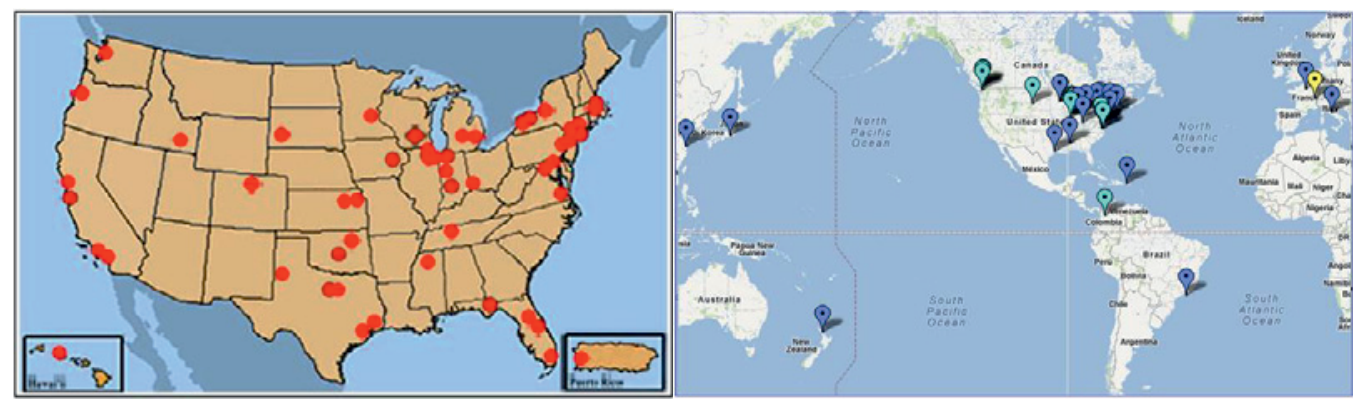

Figure 6. Maps of U.S. QuarkNet centers (left) and QuarkNet masterclass collaborators (right) indicate the reach of the QuarkNet collaboration. 


\subsubsection{QuarkNet Learning Communities}

QuarkNet centers strive to be learning communities in several important ways. Teachers work together and act as a "physics alliance" to share resources and techniques to improve their teaching. Physicists create opportunities for the teachers to learn new physics and at the same time often learn new ways to present physics from the teachers. Centers host annual workshops designed to meet local interests and needs. The program may be facilitated by QuarkNet staff and other QuarkNet teachers or completely home-grown. Some centers engage students in summer research experiences.

An example of a QuarkNet center as learning community is the center at Purdue University. In the past several years, the teachers have been active in CMS masterclasses and in using the QuarkNet cosmic ray detector to do investigations. They have piloted CMS Data Workshops, which give teachers a chance to learn more about the CMS Masterclass and bring CMS data to their students in multiple formats tailored to the schedules in their schools. The mentor, Matthew Jones, developed a new program for taking cosmic ray data from a detector. The Physics Department and QuarkNet teachers made a triple balloon flight to commemorate the $100^{\text {th }}$ anniversary of the historic flight of Victor Hess, taking cosmic ray flux data at various altitudes [10]. In summer of 2013, they participated in a CMS e-Lab workshop where they learned to analyse data beyond the masterclass [11].

\subsection{Data Portfolio and Opportunities}

\subsubsection{QuarkNet cosmic ray detector and e-Lab}

From the beginning of the QuarkNet collaboration in 1999, physicists and teachers showed great interest in building and using cosmic ray detectors as a way to make direct measurements. The first QuarkNet detectors used surplus photomultiplier tubes and scintillator along with borrowed logic crates to make muon measurements. Based on the success of these early detectors, the QuarkNet Principal Investigators and staff invested time and funds to develop and provide reliable, useful detector kits to QuarkNet students and teachers. Over 12 years of development, the detectors have evolved into the present form with a 4-channel data acquisition board that connects to a computer via USB, a GPS unit for location and nanosecond timing, and four compact counters. In workshops, students and teachers build a detector from the kit, do initial calibration, and take data in less than a day. On following days, participants learn to use the Cosmic Ray e-Lab (See below) to upload and analyse data and publish results of their investigation. The detector is used in almost every QuarkNet center-usually at multiple schools-and physicists in many countries have purchased the DAQ for other programs. In addition, the ILC collaboration has sponsored workshops and has placed many more in various locations as part of their international outreach. There are QuarkNet cosmic ray detectors or DAQ boards in India, Georgia, Russia, Israel, Poland, Germany, France, Ecuador, Mexico, Canada, Japan, Korea, Taiwan, China, and Thailand.
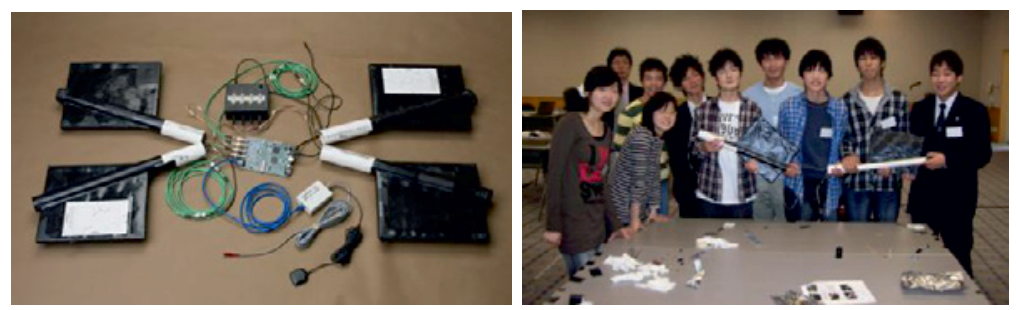

Figure 7. QuarkNet cosmic ray detector (left) and students in Tsukuba, Japan, with two counters they built.

A companion to the QuarkNet cosmic ray detector is the Cosmic Ray e-Lab [12], an online platform where students and teachers can: 


\section{EPJ Web of Conferences}

- Upload their data from the QuarkNet cosmic ray detector,

- Test the quality of their data and any other data uploaded to the e-Lab,

- Analyse their data or any data uploaded to the e-Lab, and

- Create or read online posters.

The Cosmic Ray e-Lab works in a web browser so it is very much platform independent. As of August 2013 there were over 1000 teacher accounts and over 2400 student research groups registered as users of the Cosmic Ray e-Lab.

The importance of the Cosmic Ray e-Lab is not only in its usefulness for storing and analysing cosmic ray data. It also is an instructional model for online analysis of particle physics data. The logical next step has been to create a similar tool to analyse LHC data.

\subsubsection{CMS e-Lab}

In the Cosmic Ray e-Lab, students and teachers can upload their own data, though it is also possible to use the e-Lab without having a detector. With an LHC e-Lab, the only possibility is to use data made available by one of the experiments. CMS has made a large amount of data available [13] for use in the CMS e-Lab [14]. Students and teachers can call up datasets, make cuts and create histogram plots. As in the Cosmic Ray e-Lab, CMS e-Lab users can put their results into online posters.

There are a variety of studies that can be performed with the CMS datasets. Among these are:

- Compare Z boson mass plots made from decays to $\mathrm{e}^{+} \mathrm{e}^{-}$with those made from decays to $\mu^{+} \mu^{-}$.

- Find multiple particle signals in 100,000 dimuon events in the $2-110 \mathrm{GeV}$ mass range.

- Isolate a "clean" J/ $\Psi$ signal from a "noisy" dataset.

- Study the nature of background events.

- Find evidence of cosmic rays in CMS dimuon data.

There are more studies that can be done and more will come online as a new data interface and additional datasets are added to the CMS e-Lab.
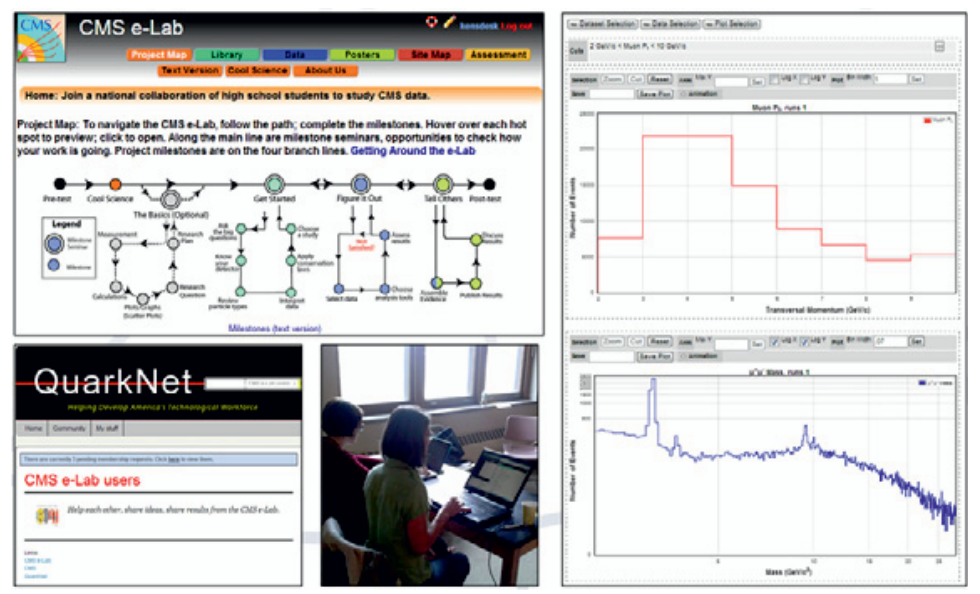

Figure 8. CMS e-Lab and its use. Clockwise from top left: CMS e-Lab project map organizes student research, transverse momentum plot for dimuon events, mass plot of 100,000 dimuon events, teachers using the CMS eLab in a workshop, and the CMS e-Lab Users Group on the QuarkNet website.

\subsubsection{Intensive Data Experiences}

QuarkNet affords teachers and students opportunities for challenging experiences with authentic data beyond activities, masterclasses and e-Labs. These are chances to engage data and physics problems in a more intensive environment. 
The flagship data experience for QuarkNet teachers has been the Particle Physics Boot Camp, a one-week workshop at Fermilab in which teachers are given csv files of 4-vectors from CMS data, links to resources, and the directive to make some sense of the data. They must learn what is in the files and, eventually, to calculate invariant masses. By the end of the week the teachers have made mass plots of the $\mathrm{Z}$ boson, the $\mathrm{J} / \Psi$ meson and the $\mathrm{W}$ boson (using transverse mass). Often they start to explore further and learn more about both the detector and what is in the data. The Boot Camp has its origin in the Lead Teacher Institutes, in which new QuarkNet teachers were introduced to particle physics through Monte Carlo data from a fictitious $\mathrm{e}^{+} \mathrm{e}^{-}$collider before CMS data was available. In 2014, the Boot Camp will become Data Camp where teachers explore more forms of LHC and other data from which to create learning units for their students.

The other intensive data experience that students and teachers can share is participation in actual research. Teachers lead teams of four students under physicist mentors for six weeks in the summer, contributing to particle physics research at various levels. At the University of Notre Dame, teachers and students have taken on such projects as:

- Deep analysis of CMS data,

- Rendering LHC hardware and data for display in a Digital Visualization Theater (similar to a planetarium),

- Experimenting with new types of scintillator, and

- CMS detector upgrade development.

\subsection{Summation}

QuarkNet is not the only program to bring particle physics data to students and teachers; indeed, QuarkNet actively collaborates with other groups, including the International Particle Physics Outreach Group (IPPOG). QuarkNet is, however, intrinsic to the overall education and outreach efforts of the U.S. particle physics community because of its long history and wide reach, its professional development and instructional methods and tools, and to its overall experience in creating opportunities for particle physics engagement.

\section{Going forward}

New opportunities and challenges await. There is room for e-Labs and masterclasses to grow in terms of usage. CMS has a new open data initiative that will make more data available from different decay processes and energies [15]. Making these classroom-ready for students and teachers will take imagination and new methods. The experience of QuarkNet CMS and ATLAS Data Workshops will be helpful: these are now being adapted to introduce groups to masterclasses in new countries. And new masterclasses from different experiments are under development. These efforts tie together in a potential revolution in physics education: bringing students and teachers to the cutting edge of physics-where the excitement is-by means of authentic data from actual research.

\section{References}

1. CMS Masterclass website, http://cms.physicsmasterclasses.org/cms.html (2013)

2. CMS website, http://cern.ch/cms

3. International Masterclasses website, http://physicsmasterclasses.org (2013)

4. CMS/Introduction to Particle Physics Workshop wiki, http://cms.michianastem.org/IPPWintro (2011)

5. CMS/Trinity School wiki, http://cms.michianastem.org/Trinity+School+May+2013 (2013)

6. U. Bilow, M. Kobel, EPJ Web of Conferences/ICNFP2013 (to be published).

7. QuarkNet website, https://quarknet.i2u2.org (2013) 
8. M. Hategan et al 2012 J. Phys.: Conf. Ser. 396 022022, http://www.i2u2.org/elab/cms/eventdisplay/ (2013)

9. CMS Collaboration, JHEP 01 (2011) 80, JHEP 10 (2011) 132

10. T. Brouck, Purdue Coll Sci Insights (http://www.science.purdue.edu/insights/spring2013/up-upand-away.html, 2013)

11. CMS e-Lab at Purdue web page, http://tinyurl.com/nzll7nv (2013)

12. QuarkNet Cosmic Ray e-Lab, http://www.i2u2.org/elab/cosmic (2013)

13. http://cms.web.cern.ch/content/cms-public-data

14. QuarkNet CMS e-Lab, http://www.i2u2.org/elab/cms (2013)

15. A. Rao, CMS Public Website, http://cms.web.cern.ch/news/lhc-data-be-made-public-open-accessinitiative (2013) 